\title{
A Functional Interleukin- 8 Haplotype Predicts Depression and Anxiety through Increased Threat-Related Amygdala Reactivity in Women but Not Men
}

\author{
Johnna R Swartz ${ }^{*}, 1,4$, Aric A Prather ${ }^{2}$, Christina R Di lorio ${ }^{3}$, Ryan Bogdan ${ }^{3}$ and Ahmad R Hariri' \\ 'Laboratory of NeuroGenetics, Department of Psychology and Neuroscience, Duke University, Durham, NC, USA; 'Department of Psychiatry, \\ University of California San Francisco, San Francisco, CA, USA; ${ }^{3}$ Department of Psychological \& Brain Sciences, Washington University in St Louis, \\ St Louis, MO, USA
}

\begin{abstract}
Common functional polymorphisms in the gene encoding interleukin- 8 (IL / 8), a cytokine belonging to the IL-I superfamily that can induce synthesis of several other cytokines, have been associated with major depressive episodes following the experience of stressful life events. The neural mechanisms underlying these associations remain unexamined. Here we use an imaging genetics strategy to examine the effects of risk-related IL I 8 haplotypes comprising rs 187238 and rs 19465 I 8 on threat-related amygdala reactivity and, through an indirect effect, stress-related symptoms of depression and anxiety in 448 non-Hispanic Caucasian university students. Analyses indicated that women but not men possessing an IL 18 haplotype comprising both risk-related alleles evidenced increased threat-related left centromedial amygdala reactivity relative to other haplotype groups. Moreover, in women only, increased threat-related left centromedial amygdala reactivity predicted increased symptoms of depression and anxiety in individuals also reporting higher levels of life stress. Path analyses revealed a significant indirect effect of $1 L 18$ risk haplotype on symptoms of depression and anxiety through increased threat-related amygdala reactivity. These results suggest that a common functional IL I 8 haplotype associated with heightened proinflammatory responses confers susceptibility to stress-related depression and anxiety through effects on threat-related amygdala function, a risk pathway specific to women. If replicated, these patterns can inform the search for personalized interventions targeting neurobiological pathways of risk associated with inflammation.
\end{abstract}

Neuropsychopharmacology (2017) 42, 419-426; doi:10.1038/npp.2016.129; published online 17 August 2016

\section{INTRODUCTION}

Individual differences in immune functioning may affect susceptibility to stress-precipitated mood and anxiety disorders. In fact, there is considerable evidence for a link between chronic inflammation and depression (Dowlati et al, 2010; Howren et al, 2009; Valkanova et al, 2013) as well as anxiety disorders (Gill et al, 2009, 2010; Passos et al, 2015). Recently, common polymorphisms in inflammation-related genes have been identified as a potential source of interindividual variability in risk for internalizing disorders (Bufalino et al, 2013). Among these candidate loci, common polymorphisms in the gene encoding interleukin-18 (IL18) have specifically been linked to the experience of major depressive episodes following stressful life events.

Prior research has demonstrated a greater frequency of riskrelated variants of IL18 promoter region polymorphisms,

\footnotetext{
*Correspondence: Dr JR Swartz, University of California, Davis, 1353 Hart Hall, Davis, CA 956I6, USA, Tel: (530) 752-3863, Fax: (530) 752-5660, E-mail: jrswartz@ucdavis.edu

${ }^{4}$ Current address: Department of Human Ecology, University of California, Davis, CA, USA.

Received 21 April 20 16; revised 12 July 2016; accepted I3 July 20 I6; accepted article preview online 19 July 2016
}

rs187238 and rs1946518, which form a haplotype, in individuals with major depressive disorder (MDD) whose illness was preceded by a stressful life event in comparison with individuals with MDD having no such experience before developing disorder (Haastrup et al, 2012), although effects would not survive correction for multiple comparisons. Moreover, an IL18 haplotype comprising the major C risk allele of rs 187238 and the major $\mathrm{G}$ risk allele of rs 1946518 has been associated with increased expression of IL-18 mRNA in peripheral blood mononuclear cells (Giedraitis et al, 2001) and plasma IL-18 (Haastrup et al, 2012). Although these prior functional and disease associations were modest and the functional associations did not reach statistical significance, they are nevertheless informative because IL-18 is a cytokine belonging to the IL-1 superfamily that can induce synthesis of several other cytokines, including tumor necrosis factor- $\alpha$ (TNF- $\alpha$ ) and IL-1 $\beta$ (Dinarello, 1999). Increased levels of IL-18 have been noted in individuals with MDD and panic disorder (Al-Hakeim et al, 2015; Kokai et al, 2002; Merendino et al, 2002; Prossin et al, 2011), and peripheral IL-18 concentrations are positively correlated with the severity of symptoms in MDD (Alcocer-Gomez et al, 2014). Moreover, post-mortem data indicate that $I L 18$ gene expression is upregulated in the 
prefrontal cortex of individuals who had MDD compared with controls (Shelton et al, 2011).

There are several potential neural mechanisms through which functional IL18 polymorphisms may influence susceptibility to stress-related disorders. One such mechanism is threat-related reactivity of the amygdala, which functions as the hub of an extended corticolimbic circuit that plays a central role in transducing signals of danger into coordinated sympathetic changes in behavior and physiology, including activation of the HPA axis (Herman and Cullinan, 1997). Importantly, IL-18 is expressed within the central nervous system and the IL-18 receptor is expressed throughout the corticolimbic circuit, including the amygdala (Alboni et al, 2009; Conti et al, 1999). Because increased expression of IL-18 can trigger the upregulation of other proinflammatory cytokines such as TNF- $\alpha$, the impact of IL18 polymorphisms on threat-related amygdala function may be mediated indirectly as well (Capuron and Miller, 2011; Miller et al, 2009).

Prior human studies provide novel evidence supporting a positive reciprocal association between amygdala reactivity and inflammation (Inagaki et al, 2012; Muscatell et al, 2015; although see Harrison et al, 2009 for contrary evidence) and an association between other inflammation-related genotypes and amygdala reactivity (see, eg, Baune et al, 2010). Importantly, heightened amygdala reactivity to social threat also predicts greater risk for the future development of mood and anxiety symptoms in response to stress (Swartz et al, 2015), raising the possibility that inflammation may confer risk for mood and anxiety disorders through its effects on amygdala reactivity. Emerging evidence also suggests that effects of inflammation may differ by sex. In one recent paper, women who were administered a low-dose endotoxin reported greater increases in depressed mood and social disconnection compared with men, even though there were no differences in the degree to which peripheral IL-6 and TNF- $\alpha$ levels increased (Moieni et al, 2015). It is possible that similar moderation by sex may occur for the association between inflammation and its effects on brain function.

Here, we use an imaging genetics strategy to test the hypothesis that IL18 haplotypes comprising rs187238 and rs1946518 risk alleles predict increased mood and anxiety symptoms through increased threat-related amygdala reactivity. We further hypothesized that these associations would be moderated by exposure to stressful life events because stress may moderate the association between the IL18 risk haplotypes and threat-related amygdala reactivity (Figure 1, path A; Sekiyama et al, 2005; Sugama and Conti, 2008). Moreover, stress may further moderate the association between amygdala reactivity and behavioral symptoms (Figure 1, path B; Swartz et al, 2015). Thus, our goal was to test both of these paths and the indirect effect from IL18 risk haplotypes on mood and anxiety symptoms. Finally, based on reported sex differences in these and related pathways, we examined moderating effects of sex (Derry et al, 2015; Moieni et al, 2015; Morris et al, 2011).

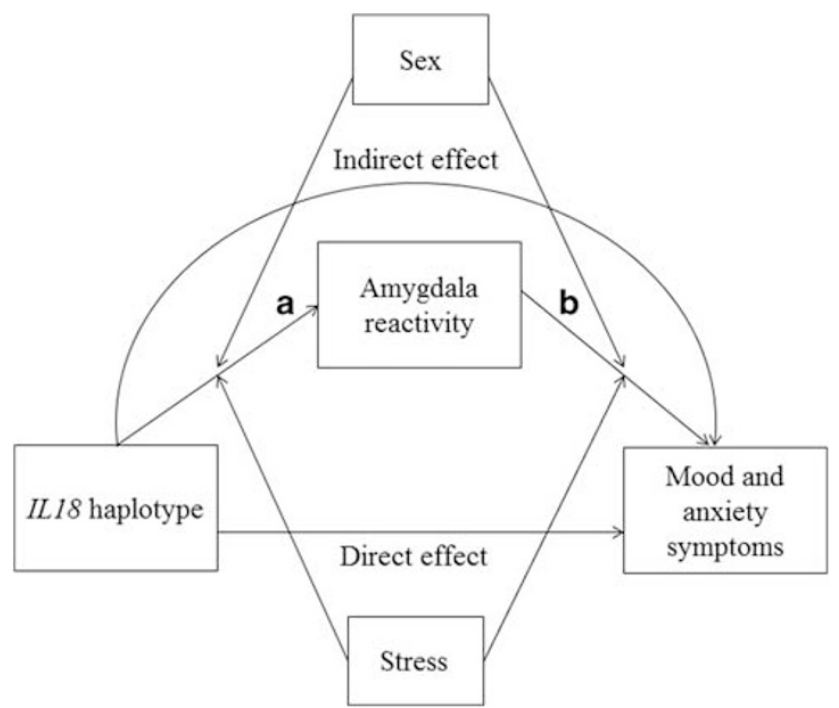

Figure I Theoretical model. Risk alleles in the ILI 8 haplotype are hypothesized to have an indirect effect on susceptibility to mood and anxiety symptoms through increased threat-related amygdala reactivity. Moderation by sex and stressful life events was tested for both paths.

\section{MATERIALS AND METHODS}

\section{Participants}

Our analyses focused on 549 young adult university students aged 18-22 years who completed the ongoing Duke Neurogenetics Study (DNS) as of December 2015 and selfreported their race and ethnicity as non-Hispanic Caucasian (Table 1). This focus was chosen to mitigate potential spurious effects due to population stratification. All procedures were approved by the Duke University Medical Center and participants provided informed consent before study initiation. Recruitment and exclusion criteria have been described in detail elsewhere (Nikolova et al, 2014; Prather et al, 2013; Swartz et al, 2015, 2016). Relatedness was assessed using pairwise identity by descent estimation in PLINK 1.07; pairs with a PI_Hat of $>0.20$ had one member excluded from analyses $(n=2)$. Diagnosis of any past or current DSMIV Axis I disorder or select Axis II disorders (antisocial personality disorder and borderline personality disorder), assessed with the electronic Mini International Neuropsychiatric Interview (Sheehan et al, 1998) and Structured Clinical Interview for the DSM-IV subtests (First et al, 1996), was not an exclusion. In the sample reported here, $22 \%$ $(n=122)$ of the participants met criteria for at least one current or past psychiatric diagnosis (see Supplementary Table S1 for specific diagnoses). However, none of the participants in the DNS were using psychotropic medications. The most commonly used medications in the sample were oral contraceptives $(n=136)$, antihistamines $(n=40)$, antibiotics $(n=18)$, bronchodilators $(n=15)$, inhaled glucocorticoids $(n=8)$, nonsteroidal anti-inflammatory drugs $(n=7)$, stimulants $(n=6)$, thyroid hormone $(n=6)$, and nasal decongestants $(n=4)$. Given that these medications may affect inflammatory pathways, we included a covariate for medication use in all analyses (further details below). 
Table I Participant Characteristics

\begin{tabular}{|c|c|c|c|}
\hline & CG/CG haplotype & Other haplotypes & Difference \\
\hline Female & $n=83$ & $n=178$ & \\
\hline Age (years) & $19.8(1.2)$ & $19.7(1.2)$ & $t(259)=-0.45, p=0.65$ \\
\hline Diagnosis & $24 \%$ & $19 \%$ & $\chi^{2}(1)=0.86, p=0.35$ \\
\hline BMI & $23.3(3.8)$ & $23.2(3.1)$ & $t(242)=-0.02, p=0.99$ \\
\hline Stressful life events & $3.0(1.3)$ & $2.9(1.3)$ & $t(259)=-0.51, p=0.61$ \\
\hline MASQ anxiety & | $8.2(5.2)$ & $17.5(5.7)$ & $t(259)=-1.02, p=0.31$ \\
\hline MASQ anxious arousal & $21.0(4.0)$ & $20.7(5.4)$ & $t(259)=-0.41, p=0.68$ \\
\hline MASQ anhedonia & $49.8(11.8)$ & $48.7(11.5)$ & $t(259)=-0.71, p=0.48$ \\
\hline MASQ total & $0.36(2.8)$ & $0.01(3.4)$ & $t(259)=-0.82, p=0.41$ \\
\hline Male & $n=79$ & $n=138$ & \\
\hline MASQ depression & $20.3(8.3)$ & $19.4(7.1)$ & $t(2 \mid 5)=-0.83, p=0.41$ \\
\hline MASQ anxiety & I7.0 (5.1) & 16.4 (4.5) & $t(2 \mid 5)=-0.88, p=0.38$ \\
\hline MASQ anxious arousal & $20.7(5.9)$ & $20.2(5.1)$ & $t(2 \mid 5)=-0.57, p=0.57$ \\
\hline MASQ anhedonia & $53.5(12.5)$ & $49.7(12.6)$ & $t(2 \mid 5)=-2.17, p=0.03$ \\
\hline MASQ total & $0.26(3.5)$ & $-0.38(3.1)$ & $t(215)=-1.40, p=0.17$ \\
\hline
\end{tabular}

Abbreviations: BMI, body mass index; MASQ, Mood and Anxiety Symptom Questionnaire.

Diagnosis indicates the percentage of participants with any past or present psychiatric diagnosis. Stressful life events indicate the summed impact scores across all life events reported on the Life Event Scale for Students (square root transformed). MASQ Total was calculated by standardizing each subscale score and summing them; a score of 0 therefore indicates mean levels of symptoms.

\section{Amygdala Reactivity Paradigm}

Amygdala reactivity to threat was assessed using an emotional face matching challenge paradigm. The paradigm version used in the DNS consists of four blocks of a face-processing task (one block each for fearful, angry, surprised, and neutral faces) interleaved with five blocks of a sensorimotor control task. Details on the task paradigm have been reported in previous research (Swartz et al, 2015, 2016).

\section{BOLD fMRI Data Acquisition, Preprocessing, and Quality Assurance}

Participants were scanned using a research-dedicated GE MR750 3T scanner at the Duke-UNC Brain Imaging and Analysis Center. A series of 34 interleaved axial functional slices aligned with the anterior commissure-posterior commissure (AC-PC) plane were acquired for full-brain coverage using an inverse-spiral pulse sequence to reduce susceptibility artifact $(\mathrm{TR}=2000 \mathrm{~ms}$; $\mathrm{TE}=30 \mathrm{~ms}$; flip angle $=$ 60 ; FOV $=240 \mathrm{~mm} ; 3.75 \times 3.75 \times 4 \mathrm{~mm}$ voxels; interslice skip $=0$ ).

Functional MRI data were processed in SPM8 using the standard preprocessing stream used in previously published research from the DNS (Nikolova et al, 2014; Prather et al, 2013; Swartz et al, 2015, 2016). Further details on preprocessing and quality control procedures are reported in the Supplementary Materials and Methods. The current sample includes 502 participants with fMRI data meeting all quality control criteria.

\section{BOLD fMRI Data Analysis}

The general linear model of SPM8 was used to conduct fMRI data analyses. Following preprocessing, a first-level model was created for each participant that included separate regressors for each block type (fearful, angry, surprised, and neutral faces and shape matching control blocks), as well as motion regressors created by artifact detection software (see Supplementary Information for further details). Next, individual contrast images for effects of each expression were generated at the first level for each participant and then entered into second-level random effects models to determine mean condition-specific regional responses. We extracted parameter estimates from functional clusters within amygdala regions of interest activated to the condition at $p<0.05$ family-wise error (FWE) corrected across the search volumes, for the contrast of Angry and Fearful blocks > Control blocks. Amygdala regions of interest were derived from probabilistic cytoarchitectonic mapping of the basolateral and centromedial amygdala subregions (Amunts et al, 2005). Analyses were first performed for the contrast of Angry and Fearful blocks > Control blocks; when significant effects were observed, post hoc analyses were conducted to determine whether effects were driven by either the Angry or Fearful blocks specifically. 


\section{Genotyping}

Genotyping was conducted by 23andMe. Genomic DNA from all participants was isolated from buccal cells derived from Oragene DNA self-collection kits (DNA Genotek, Kanata, Canada) customized for 23andMe. DNA extraction and genotyping were performed at the National Genetics Institute, a CLIA-certified clinical laboratory and subsidiary of Laboratory Corporation of America. One of two different Illumina arrays with custom content was used to provide genome-wide SNP data, the HumanOmniExpress or HumanOmniExpress-24 (Do et al, 2011; Eriksson et al, 2010; Tung et al, 2011). Both rs187238 and rs1946518 were genotyped on these arrays. Genotype distribution did not deviate from Hardy-Weinberg equilibrium for $\operatorname{rs} 187238\left(\chi^{2}(1)=1.58, p=0.21\right)$ or for rs 1946518 $\left(\chi^{2}(1)=0.004, p=0.95\right)$. PHASE was used to generate IL18 haplotypes (Stephens et al, 2001); in all cases posterior probability was $>99 \%$. Based on prior research (Giedraitis et al, 2001; Haastrup et al, 2012), we coded individuals homozygous for the CG haplotype (ie, homozygous for the major C allele of rs187238 and for the major G allele of rs1946518) as the group of interest $(n=162)$ and all other individuals as the reference group $(n=316)$. See the Supplementary Materials and Methods for additional analyses supporting this grouping. Genotype data were available in 478 participants (448 of whom had valid fMRI data).

\section{Stress}

Concurrent to the collection of genotype and fMRI data, recent life stress was assessed with the Life Events Scale for Students (LESS; Clements and Turpin, 1996). The LESS is a checklist of life events; for each event, participants reported whether or not that event had occurred in the past year and rated the severity of the impact of that event on a 1 to 4 scale $(4=$ severe impact). Similar to our prior work (Swartz et al, 2015), we summed the severity ratings for each event present to create a total impact score, reflecting both a greater number and severity of life events. Because this variable was skewed, a square root transformation was applied to yield a more normal distribution.

\section{Mood and Anxiety Symptoms}

Concurrent to the collection of genotype, fMRI, and stress data, participants reported on their mood and anxiety symptoms with the Mood and Anxiety Symptoms Questionnaire (MASQ) Short Form (Watson et al, 1995). The MASQ consists of four subscales including general depression, general anxiety, anxious arousal, and anhedonia. Participants were asked to report on symptoms they experienced in the past week (eg, 'Felt worthless') on a scale from 1 (not at all) to 5 (extremely). Total scores on the MASQ were calculated by standardizing scores on the four subscales and then summing scores. When significant effects were detected with MASQ total scores, post hoc analyses were conducted to examine whether they were driven by specific subscales of the MASQ.

\section{Covariates}

Higher body mass index (BMI) is associated with greater proinflammatory response to social stress (McInnis et al, 2014). Therefore, BMI was calculated using participants' height and weight and was entered as a covariate in all analyses. BMI was missing for 44 participants. To control for potential circadian variation in amygdala reactivity and inflammation, we also entered time of day for the scan as a covariate in all analyses (range: 0800 to $1900 \mathrm{~h}$ ). Psychiatric diagnoses (both past and current) were dummy-coded as mood and anxiety disorders $(n=61)$ or other psychopathology, generally alcohol and substance use disorders $(n=61)$; in the case of comorbid diagnoses, they were included in the mood and anxiety disorder group. These two dummycoded variables were entered as covariates in all analyses. A covariate was also created for medication use $(0=$ no medication, $1=$ medication), including all classes of medications other than oral contraceptives. In addition, to control for ancestral genetic heterogeneity within the self-reported non-Hispanic Caucasian participants, we computed multidimensional scaling (MDS) components using identity-bystate (IBS) analyses in PLINK of the whole genome data within this subgroup. We included the top two MDS components as covariates in all of our statistical models. Additional supplementary analyses were conducted in women only controlling for oral contraceptive use and phase of menstrual cycle (see Supplementary Information).

\section{Statistical Analysis}

All analyses were conducted in Mplus v7 (Muthen \& Muthen) using full information maximum likelihood estimation (FIML). For all analyses, 1000 bootstrapped samples were requested to generate standard errors robust to nonnormality. Our goal was to build an indirect effects model linking IL18 haplotype to mood and anxiety symptoms via threat-related amygdala reactivity (Figure 1). To do so, we tested each path separately first, and then used results from these analyses to construct the full model. Full details on the analytical approach are reported in the Supplementary Materials and Methods.

\section{RESULTS}

\section{Effects of IL18 Haplotype on Amygdala Reactivity}

The omnibus test was significant $\left(\Delta \chi^{2}(7)=20.60, p=0.004\right)$ indicating that the effects of IL18 haplotype on amygdala reactivity differed by sex and/or amygdala subregion. Followup analyses to test for interactions between haplotype and sex across the four subregions indicated that sex significantly moderated the effect of IL18 haplotype on left centromedial amygdala reactivity $\left(\Delta \chi^{2}(1)=6.73, p=0.01\right.$, false discovery rate (FDR)-corrected $p=0.04)$. This was because of a significant effect of the haplotype on amygdala reactivity in women $\left(\mathrm{B}=0.07, \mathrm{SE}=0.03, \beta=0.17, p=0.01, \Delta R^{2}=0.03\right)$ but not in men $(\mathrm{B}=-0.04, \mathrm{SE}=0.04, \beta=-0.09, p=0.24$, $\Delta R^{2}=0.01$; Figure 2). The effect also remained significant in women when winsorizing continuous variables to limit the influence of outliers. Further post hoc testing indicated that the effect in women was specific to the left centromedial 


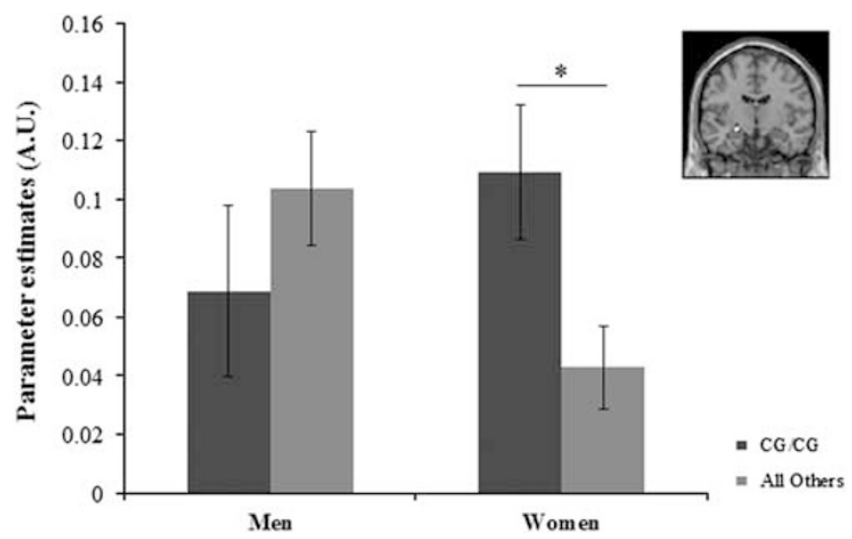

Figure 2 The association between IL/8 haplotype and amygdala reactivity is moderated by sex. Parameter estimates were extracted for the contrast of Angry and Fearful Faces $>$ Control blocks within a functional cluster significantly activated at $p<0.05$ family-wise error-corrected within the left centromedial amygdala (inset). IL I 8 haplotype predicted significant differences in left centromedial amygdala reactivity in women but not men. CG/CG haplotype group indicates participants homozygous for the rs 187238 major $C$ allele and for the rs 19465 I 8 major $G$ allele. Error bars represent I SEM; *p $<0.05$.

amygdala, as parameter estimates differed significantly for this region compared with the left basolateral $\left(\Delta \chi^{2}(1)=8.09\right.$, $p=0.004$, FDR-corrected $p=0.012)$, the right centromedial $\left(\Delta \chi^{2}(1)=5.26, p=0.02\right.$, FDR-corrected $\left.p=0.02\right)$, and the right basolateral amygdala $\left(\Delta \chi^{2}(1)=5.20, p=0.02\right.$, FDRcorrected $p=0.02$ ). The post hoc testing also indicated this effect did not differ by facial expression $\left(\Delta \chi^{2}(1)=0.07\right.$, $p=0.79)$; in other words, in women the effect of $I L 18$ haplotype was equivalent for left centromedial amygdala reactivity to angry and fearful facial expressions. Moderating effects of sex and main effects of haplotype were not significant for the other subregions tested (all $p$ 's $>0.05$ ). See Supplementary Materials and Methods for further analyses supporting these results.

\section{Moderation of the Effect of IL18 Haplotype by Recent Life Stress}

The omnibus test indicated that there were no significant IL18 haplotype $\times$ stress interaction effects for the four amygdala subregions in either men or women $\left(\Delta \chi^{2}(8)=4.65\right.$, $p=0.79$ ). Looking just at the left centromedial amygdala in women, when the IL18 haplotype $\times$ stress interaction was entered into the regression, the main effect of IL18 haplotype remained significant $(p=0.012)$, but the interaction of haplotype $\times$ stress was not significant $(B=-0.03, S E=0.02$, $\beta=-0.10, p=0.16$ ).

\section{Effect of Amygdala Reactivity on Concurrent Mood and Anxiety Symptoms}

Because the effects of the IL18 haplotype were only significant for left centromedial amygdala reactivity, we examined the interaction of this subregion and recent stress as a predictor of concurrent mood and anxiety symptoms. Multigroup analyses indicated that the main effect of left centromedial amygdala reactivity on MASQ total symptoms

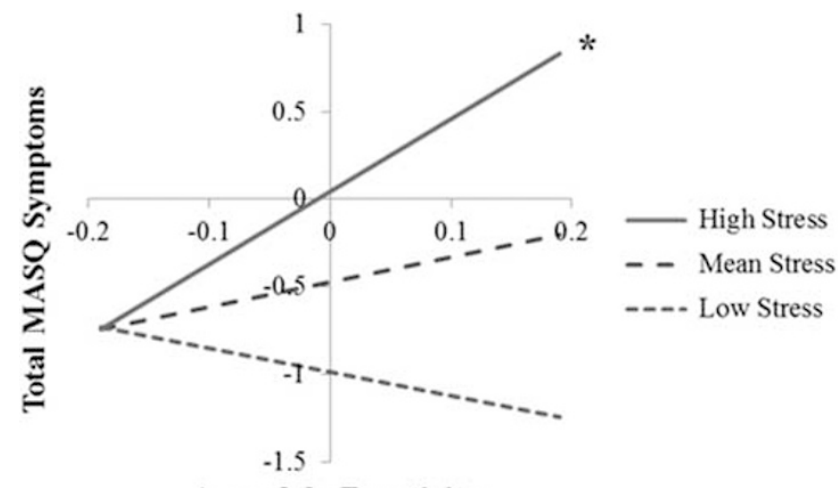

Amygdala Reactivity

Figure 3 Interaction between left centromedial amygdala reactivity and recent stress predicts concurrent mood and anxiety symptoms in women. Effects are shown for women only. Simple slopes analysis was conducted using parameter estimates obtained in Mplus. Effects were estimated at mean levels of stress, I SD below the mean (low stress) and I SD above the mean (high stress). The symbol '*' indicates that a simple slope is significant at $p<0.05$. Amygdala reactivity was mean centered for this analysis. Total MASQ symptoms were calculated by summing the four standardized subscale scores; 0 indicates mean symptom levels across the subscales.

was not significantly moderated by sex $(p=0.71)$ and was not significant $(\mathrm{B}=1.07, \mathrm{SE}=0.76, p=0.16)$. However, multigroup analyses indicated the interaction between amygdala reactivity and stress in predicting symptoms was significantly moderated by sex $\left(\Delta \chi^{2}(1)=5.84, p=0.02\right)$. In women, there was a significant interaction between left centromedial amygdala reactivity and stress in predicting total MASQ symptoms $(\mathrm{B}=2.08, \quad \mathrm{SE}=0.79, \quad \beta=0.16$, $\left.p=0.01, \Delta R^{2}=0.02\right)$. As demonstrated in Figure 3 , greater amygdala reactivity predicted greater mood and anxiety symptoms, but only under conditions of higher stress. This interaction was not significant in men $(B=-0.45, S E=1.11$, $\left.\beta=-0.04, p=0.69, \Delta R^{2}=0.001\right)$. The effect also remained significant in women when winsorizing continuous variables to limit the influence of outliers. The post hoc analyses indicated that this interaction effect was equivalent for amygdala reactivity to fearful and angry faces $\left(\Delta \chi^{2}(1)=0.02\right.$, $p=0.89$ ). The post hoc analyses also indicated this interaction was significant in women for the subscales of depression $\left(\mathrm{B}=5.00, \mathrm{SE}=1.82, \beta=0.16, p=0.006, \Delta R^{2}=0.02\right)$, and marginally anxiety $(\mathrm{B}=2.85, \mathrm{SE}=1.57, \beta=0.13, p=0.07$, $\left.\Delta R^{2}=0.02\right)$, anxious arousal $(\mathrm{B}=2.99, \mathrm{SE}=1.51, \beta=0.15$, $\left.p=0.05, \Delta R^{2}=0.02\right)$, and anhedonia $(\mathrm{B}=3.13, \mathrm{SE}=2.58$, $\left.\beta=0.07, p=0.23, \Delta R^{2}=0.004\right)$. Constraining parameter estimates to be equal across the four subscales did not result in a significant reduction in model fit $\left(\Delta \chi^{2}(3)=2.86\right.$, $p=0.41$ ), indicating that effects were generally equivalent across the four subscales.

\section{Indirect Effect of IL18 Haplotype on Symptoms via Amygdala Reactivity}

Based on the results of the analyses above, we report an indirect effects model in women only, as neither path was significant in men. On the first path (Figure 1, path A) we modeled the main effect of IL18 haplotype on left centromedial amygdala reactivity, given no evidence for 
moderation by recent life stress. On the second path (Figure 1, path B), we modeled an interaction between recent life stress and left centromedial amygdala reactivity as a predictor of total MASQ symptoms. As shown in Figure 4, there was a significant indirect effect of IL18 haplotype on mood and anxiety symptoms through its effect on threatrelated amygdala reactivity. As expected, this indirect effect was significant when stress was $1 \mathrm{SD}$ above the mean $(\alpha \beta=0.29, \mathrm{SE}=0.14, p=0.04,95 \%$ confidence intervals $(0.08$ to 0.67$)$ ), but not when stress was at mean levels $(\alpha \beta=0.10$, $\mathrm{SE}=0.08, p=0.22(-0.03$ to 0.30$))$ or $1 \mathrm{SD}$ below the mean $(\alpha \beta=-0.09, \mathrm{SE}=0.13, p=0.50,(-0.43$ to 0.10$))$.

\section{DISCUSSION}

The goal of the present study was to examine whether an IL18 risk-related functional haplotype predicted individual differences in threat-related amygdala reactivity and, indirectly, mood and anxiety symptoms as a function of stress. We found that in women IL18 haplotype predicted relatively increased threat-related reactivity of the left centromedial amygdala. We also found an indirect effect of IL18 haplotype on mood and anxiety symptoms under conditions of higher stress via its effect on amygdala reactivity.

We found that the effects of the IL18 haplotype were strongest for the left centromedial amygdala subregion. This is notable as the centromedial subregion encompasses the central nucleus of the amygdala (CeA), which facilitates activation of the HPA axis in response to threat via projections to the paraventricular nucleus. The specificity of this effect to women aligns with the finding that acute activation of the inflammatory response through administration of lowdose endotoxin results in greater reductions in mood in women compared with men (Moieni et al, 2015). Future research will be necessary to confirm these sex differences in genetic influences on amygdala reactivity and to examine whether the IL18 haplotype may affect functioning of regions other than the amygdala in men. Indeed, prior research has indicated that the structure, function, and connectivity of other regions implicated in internalizing disorders, including the hippocampus, ventral striatum, and prefrontal cortex, are associated with individual differences in proinflammatory markers and inflammation-related genotypes (Baune et al, 2012; Inagaki et al, 2015; Muscatell et al, 2015), suggesting that these regions should also be investigated as potential mediators of IL18 haplotype effects on stress-related internalizing symptoms.

The molecular and cellular mechanisms driving the association between IL18 haplotype and threat-related amygdala reactivity remain to be determined. Because IL-18 is expressed in the brain (Conti et al, 1999) and IL-18 receptors are present in the amygdala (Alboni et al, 2009), direct modulatory effects on threat-related reactivity are possible. As discussed earlier, IL-18 can also drive the production of other proinflammatory cytokines including IL-6 and IL-1 $\beta$ (Dinarello, 1999) that may affect brain function through a number of parallel pathways (Capuron and Miller, 2011). In another line of work in MDD, sadnessinduced increases in IL-18 have been associated with sadness-induced endogenous opioid release in the left amygdala that was associated with changes in negative

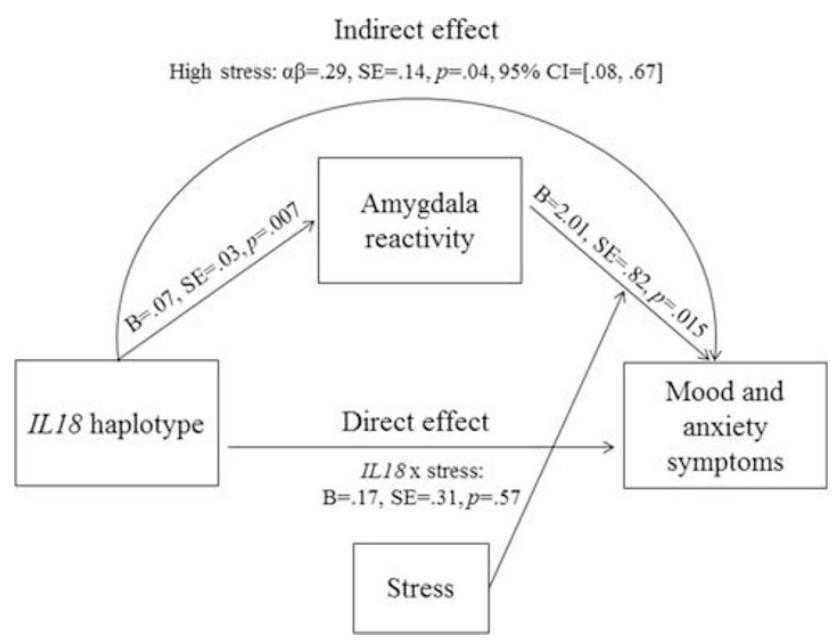

Figure 4 Indirect effect of IL/8 haplotype on mood and anxiety symptoms via amygdala reactivity in women. Indirect effect model is shown for women only; model is not shown for men because neither path was significant. The CG/CG IL 8 haplotype group (individuals homozygous for the rs/87238 major $C$ allele and for the rs19465।8 major $G$ allele) evidenced increased left centromedial threat-related amygdala reactivity. Increased threat-related amygdala reactivity in turn predicted higher mood and anxiety symptoms under higher levels of recent life stress. The indirect effect of IL I 8 haplotype on mood and anxiety symptoms through amygdala reactivity was significant, but only in individuals reporting higher levels of life stress.

affective state (Prossin et al, 2016). Animal models also indicate that IL-18 may affect expression of a wide range of genes in the amygdala, with IL18 knockout mice evidencing altered expression of over 1000 genes in the amygdala, including those encoding neuropeptides known to regulate threat-related amygdala reactivity such as oxytocin and arginine vasopressin (Yamamoto et al, 2010). Thus, there are many potential routes through which IL-18 may affect amygdala reactivity and broader corticolimbic circuit function. Counter to expectations, we did not observe that the association between IL18 haplotype and threat-related amygdala reactivity was moderated by stressful life events. We might have observed stronger effects with a more acute measure of life stress (ie, life stress within the past week or the past month) than our measure of life stress within the past year, or it may be that our measure of stressful life events was too broad and that moderation may have been observed by focusing on specific types of stressors. Moreover, stronger moderating effects may be observed for more chronic and severe forms of stress, such as childhood maltreatment (Redlich et al, 2015).

Several limitations of the present study warrant discussion. First, we did not obtain measures of peripheral IL-18 in participants, and hence we were unable to test the effect of IL18 haplotype on peripheral inflammation, or whether effects of the haplotype on amygdala reactivity were mediated by higher peripheral IL-18 levels. This is an important direction for future research. Second, we did not have a more acute measure of recent life stress (ie, within the past week, which may have reduced our ability to detect a moderating effect of life stress on the association between IL18 haplotype and amygdala reactivity. Third, because a relatively small proportion of participants reported a current 
mood or anxiety disorder $(n=29)$, we did not test whether threat-related amygdala reactivity predicted clinical diagnoses. Thus, it remains to be determined whether the associations observed with a continuous distribution of symptoms also apply when examining clinically significant categorical disorder. Fourth, because this study is crosssectional, temporal order of the relationship between the amygdala by stressful life event interaction and mood and anxiety symptoms cannot be established. However, our prior work showing that this interaction precedes the development of prospectively assessed mood symptoms is consistent with our model (Swartz et al, 2015).

These limitations notwithstanding, we provide initial evidence that a functional IL18 haplotype indirectly influences stress-related susceptibility to mood and anxiety symptoms through heightened threat-related amygdala reactivity, an effect observed only in women. As such, our results inform ongoing efforts to devise genetic risk profiles to identify specific pathophysiological mechanisms contributing to stress susceptibility in individuals and, ultimately, using personalized approaches to target preventions or interventions toward these pathways of risk.

\section{FUNDING AND DISCLOSURE}

AAP has received compensation for consulting from Posit Science on a project unrelated to this research. JRS, CRDI, $\mathrm{RB}$, and ARH declare no conflict of interest. The Duke Neurogenetics Study is supported by Duke University and NIH Grant DA033369. JRS received support through the Center for the Study of Adolescent Risk and Resilience (P30DA023026) and through NIH Grant R01AG049789. AAP is supported by NIH Grant K08HL112961. RB is supported by the Klingenstein Third Generation Foundation and NIH Grant AG045231. ARH is supported by NIH Grants R01DA033369 and R01AG049789.

\section{ACKNOWLEDGMENTS}

We thank Annchen Knodt for assistance with genetic and fMRI data processing and Spenser Radtke for assistance with data collection.

\section{REFERENCES}

Al-Hakeim HK, Al-Rammahi DA, Al-Dujaili AH (2015). IL-6, IL-18, sIL-2R, and TNF- $\alpha$ proinflammatory markers in depression and schizophrenia patients who are free of overt inflammation. J Affect Disord 182: 106-114.

Alboni S, Cervia D, Ross B, Montanari C, Gonzalez AS, SanchezAlavez $M$ et al (2009). Mapping of the full length and the truncated interleukin-18 receptor alpha in the mouse brain. J Neuroimmunol 214: 43-54.

Alcocer-Gomez E, De Miguel M, Casas-Barquero N, Nunez-Vasco J, Sanchez-Alcazar JA, Fernandez-Rodriguez A et al (2014). NLRP3 inflammasome is activated in mononuclear blood cells from patients with major depressive disorder. Brain Behav Immun 36: 111-117.

Amunts K, Kedo O, Kindler M, Pieperhoff P, Mohlberg H, Shah N et al (2005). Cytoarchitectonic mapping of the human amygdala, hippocampal region and entorhinal cortex: intersubject variability and probability maps. Anat Embryol (Berl) 210: 343-352.
Baune BT, Dannlowski U, Domschke K, Janssen DGA, Jordan MA, Ohrmann P et al (2010). The interleukin 1 beta (IL1 $\beta$ ) gene is associated with failure to achieve remission and impaired emotion processing in major depression. Biol Psychiatry 67: 543-549.

Baune BT, Konrad C, Grotegerd D, Suslow T, Birosova E, Ohrmann P et al (2012). Interleukin-6 gene (IL-6): A possible role in brain morphology in the healthy adult brain. J Neuroinflamm 9: 125.

Bufalino C, Hepgul N, Aguglia E, Pariante CM (2013). The role of immune genes in the association between depression and inflammation: a review of recent clinical studies. Brain Behav Immun 31: 31-47.

Capuron L, Miller AH (2011). Immune system to brain signaling: neuropsychopharmacological implications. Pharmacol Ther 130: $226-238$

Clements K, Turpin G (1996). The life events scale for students: validation for use with British samples. Pers Individ Differ 20: 747-751.

Conti B, Park LCH, Calingasan NY, Kim Y, Kim H, Bae Y et al (1999). Cultures of astrocytes and microglia express interleukin 18. Mol Brain Res 67: 46-52.

Derry HM, Padin AC, Kuo JL, Hughes S, Kiecolt-Glaser J (2015). Sex differences in depression: does inflammation play a role? Curr Psychiatry Rep 17: 78.

Dinarello CA (1999). IL-18: a TH1-inducing, proinflammatory cytokine and new member of the IL-1 family. I Allergy Clin Immunol 103: 11-24.

Do CB, Tung JY, Dorfman E, Kiefer AK, Drabant EM, Francke U et al (2011). Web-based genome-wide association study identifies two novel loci and a substantial genetic component for Parkinson's disease. PLoS Genet 7: e1002141.

Dowlati Y, Herrmann N, Swardfager W, Liu H, Sham L, Reim EK et al (2010). A meta-analysis of cytokines in major depression. Biol Psychiatry 67: 446-457.

Eriksson N, Macpherson JM, Tung JY, Hon LS, Naughton B, Saxonov S et al (2010). Web-based, participant-driven studies yield novel genetic associations for common traits. PLoS Genet 6: e1000993.

First MB, Spitzer RL, Gibbon M, Williams JBM (1996). Structured Clinical Interview for DSM-IV Axis I Disorders Research Version. Non-Patient Edition. New York State Psychiatric Institute, Biometrics Research Department: New York.

Giedraitis V, He B, Huang W, Hillert J (2001). Cloning and mutation analysis of the human IL-18 promoter: a possible role of polymorphisms in expression regulation. J Neuroimmunol 112: 146-152.

Gill JM, Saligan L, Woods S, Page G (2009). PTSD is associated with an excess of inflammatory immune activities. Perspect Psychiatr Care 45: 262-277.

Gill JM, Vythilingam M, Page GG (2010). Low cortisol, high DHEA, and high levels of stimulated TNF- $\alpha$, and IL- 6 in women with PTSD. J Trauma Stress 21: 530-539.

Haastrup E, Bukh JD, Bock C, Vinberg M, Thorner LW, Hansen T et al (2012). Promoter variants in IL18 are associated with onset of depression in patients previously exposed to stressfullife events. J Affect Disord 136: 134-138.

Harrison NA, Byrdon L, Walker C, Gray MA, Steptoe A, Critchley HD (2009). Inflammation causes mood changes through alterations in subgenual cingulate activity and mesolimbic connectivity. Biol Psychiatry 66: 407-414.

Herman JP, Cullinan WE (1997). Neurocircuitry of stress: central control of the hypothalamo-pituitary-adrenocortical axis. Trends Neurosci 20: 78-84.

Howren MB, Lamkin DM, Suls J (2009). Associations of depression with C-reactive protein, IL-1, and IL-6: a meta-analysis. Psychosom Med 71: 171-186.

Inagaki TK, Muscatell KA, Irwin MR, Cole SW, Eisenberger NI (2012). Inflammation selectively enhances amygdala activity to socially threatening images. Neuroimage 59: 3222-3226. 
Inagaki TK, Muscatell KA, Irwin MR, Moieni M, Dutcher JM, Jevtic I et al (2015). The role of the ventral striatum in inflammatoryinduced approach toward support figures. Brain Behav Immun 44: 247-252.

Kokai M, Kashiwamura S, Okamura H, Ohara K, Morita Y (2002). Plasma interleukin-18 levels in patients with psychiatric disorders. J Immunother 25: S68-S71.

McInnis CM, Thoma MV, Gianferante D, Hanlin L, Chen X, Breines JG et al (2014). Measures of adiposity predict interleukin6 responses to repeated psychosocial stress. Brain Behav Immun 42: 33-40.

Merendino RA, Di Rosa AE, Di Pasquale G, Minciullo PL, Mangraviti C, Costantino A et al (2002). Interleukin-18 and CD30 serum levels in patients with moderate-severe depression. Mediators Inflamm 11: 265-267.

Miller AH, Maletic V, Raison CL (2009). Inflammation and its discontents: the role of cytokines in the pathophysiology of major depression. Biol Psychiatry 65: 732-741.

Moieni M, Irwin MR, Jevtic I, Olmstead R, Breen EC, Eisenberger NI (2015). Sex differences in depressive and socioemotional responses to an inflammatory challenge: implications for sex differences in depression. Neuropsychopharmacology 40: 1709-1716.

Morris AA, Zhao L, Ahmed Y, Stoyanova N, De Staercke C, Hooper WC et al (2011). Association between depression and inflammation-differences by race and sex: the METAHealth Study. Psychosom Med 73: 462-468.

Muscatell KA, Dedovic K, Slavich GM, Jarcho MR, Breen EC, Bower JE et al (2015). Greater amygdala activity and dorsomedial prefrontal-amygdala coupling are associated with enhanced inflammtory responses to stress. Brain Behav Immun 43: 46-53.

Nikolova YS, Koenen KC, Galea S, Wang C, Seney ML, Sibille E et al (2014). Beyond genotype: serotonin transporter epigenetic modification predicts human brain function. Nat Neurosci 17: 1153-1155.

Passos IC, Vasconcelos-Moreno M, Costa L, Kunz M, Brietzke E, Quevedo J et al (2015). Inflammatory markers in post-traumatic stress disorder: a systematic review, meta-analysis, and metaregression. Lancet Psychiatry 2: 1002-1012.

Prather AA, Bogdan R, Hariri AR (2013). Impact of sleep quality on amygdala reactivity, negative affect, and perceived stress. Psychosom Med 75: 350-358.

Prossin AR, Koch AE, Campbell PL, Barichello T, Zalcman SS, Zubieta JK (2016). Acute experimental changes in mood state regulate immune function in relation to central opioid neurotransmission: a model of human CNS-peripheral inflammatory interaction. Mol Psychiatry 21: 243-251.

Prossin AR, Koch AE, Campbell PL, McInnis MG, Zalcman SS, Zubieta JK (2011). Association of plasma interleukin-18 levels with emotion regulation and $\mu$-opioid neurotransmitter function in major depression and healthy volunteers. Biol Psychiatry 69: 808-812.

Redlich R, Stacey D, Opel N, Grotegerd D, Dohm K, Kugel H et al (2015). Evidence of an IFN- $\gamma$ by early life stress interaction in the regulation of amygdala reactivity to emotional stimuli. Psychoneuroendocrinology 62: 166-173.

Sekiyama A, Ueda H, Kashiwamura S, Sekiyama R, Takeda M, Rokutan $\mathrm{K}$ et al (2005). A stress-induced, superoxide-mediated caspase-1 activation pathway causes plasma IL-18 upregulation. Immunity 22: 669-677.

Sheehan DV, Lecrubier Y, Sheehan KH, Amorim P, Janavs J, Weiller E et al (1998). The Mini-International Neuropsychiatric Interview (M.I.N.I.): the development and validation of a structured diagnostic psychiatric interview for DSM-IV and ICD-10. J Clin Psychiatry 59: 22-33.

Shelton RC, Claiborne J, Sidoryk-Wegrzynowicz M, Reddy R, Aschner M, Lewis DA et al (2011). Altered expression of genes involved in inflammation and apoptosis in frontal cortex in major depression. Mol Psychiatry 16: 751-762.

Stephens M, Smith NJ, Donnelly P (2001). A new statistical method for haplotype reconstruction from population data. Am J Hum Genet 68: 978-989.

Sugama S, Conti B (2008). Interleukin-18 and stress. Brain Res Rev 58: 85-95.

Swartz JR, Knodt AR, Radtke SR, Hariri AR (2015). A neural biomarker of psychological vulnerability to future life stress. Neuron 85: 505-511.

Swartz JR, Waller R, Bogdan R, Knodt AR, Sabhlok A, Hyde LW et al (2016). A common polymorphism in a Williams syndrome gene predicts amygdala reactivity and extraversion in healthy adults. Biol Psychiatry (e-pub ahead of print 15 December 2015; doi:10.1016/j.biopsych.2015.12.007).

Tung JY, Do CB, Hinds DA, Kiefer AK, Macpherson JM, Chowdry $\mathrm{AB}$ et al (2011). Efficient replication of over 180 genetic associations with self-reported medical data. PLoS One 6: e23473.

Valkanova V, Ebmeier KP, Allan CL (2013). CRP, IL-6 and depression: a systematic review and meta-analysis of longitudinal studies. J Affect Disord 150: 736-744.

Watson D, Clark LA, Weber K, Assenheimer JS, Strauss ME, McCormick RA (1995). Testing a tripartite model: II. Exploring the symptom structure of anxiety and depression in student, adult, and patient samples. J Abnorm Psychol 104: 15-25.

Yamamoto Y, Tanahashi T, Katsuura S, Kurokawa K, Nishida K, Kuwano Y et al (2010). Interleukin-18 deficiency reduces neuropeptide gene expressions in the mouse amygdala related with behavioral change. J Neuroimmunol 229: 129-139.

Supplementary Information accompanies the paper on the Neuropsychopharmacology website (http://www.nature.com/npp) 